\title{
Modelo compacto con capacidad de predicción de parámetros físicos para amplificadores de RF
}

Fecha de recepción: 18 de enero de 2019

Guillermo Rafael-Valdivia ${ }^{1}$

Fecha de aprobación: 29 de marzo de 2019

\section{Resumen}

En el presente trabajo se ha presentado un análisis de transistores de efecto de campo usando fuentes de voltaje pulsadas. Se han realizado medidas de microondas en dispositivos de tecnología HEMT's y LDMOS poniendo en evidencia la diferencia entre el comportamiento estático y dinámico de dichos dispositivos. En base a las medidas se ha realizado un procesamiento de datos derivando una nueva ecuación con la capacidad de reproducir ambos tipos de comportamiento con elevada precisión y en diferentes puntos de operación. Consecuentemente el trabajo aporta un nuevo modelo basado en un circuito no lineal de cuatro terminales. La relevancia de este modelo es la capacidad de predecir los efectos físicos como la dispersión frecuencial y la movilidad electrónica del dispositivo semiconductor. Esto es importante pues la dispersión frecuencial es uno de los problemas más importantes de los sistemas de comunicación modernos que genera efectos memoria limitando la capacidad de transmitir señales de gran ancho de banda. El hecho de poder predecir la movilidad electrónica y la dispersión frecuencial ayudan al diseñador de circuitos a mejorar su calidad y tiempo de diseño. Además, permite a la industria de fabricación de componentes de RF ahorrar costos de producción pues esta técnica permite predecir el comportamiento de los circuitos antes de implementarlos. La metodología para la obtención del modelo compacto ha sido validada a través de la implementación de un amplificador de potencia en tecnología LDMOS usando la técnica propuesta. El modelo propuesto es abierto puesto que la nueva técnica propuesta se puede implementar en cualquiera de los modelos convencionales usados actualmente en el ámbito industrial y académico.

Palabras clave: amplificadores de potencia RF; GaAs; microondas; movilidad electrónica; transistores de efecto de campo.

\section{Compact model with physical parameter prediction capability for RF amplifiers}

\begin{abstract}
In this work presents an analysis of field effect transistors using pulsed voltage sources has been presented. Microwave measurements have been made in HEMT's and LDMOS technology devices highlighting the difference between the static and dynamic behavior of these devices. Based on measurements, data processing has been performed, deriving a new equation with the ability to reproduce both types of behavior with high precision and at different points of operation. Consequently, the work provides a new model based on a non-linear four-terminal circuit. The relevance of this model is
\end{abstract}

${ }^{1}$ Ph. D. Universidad Tecnológica del Perú (Arequipa, Perú). c16699@utp.edu.pe. ORCID: 0000-0001-8855$\underline{6641}$.

Revista Facultad de Ingeniería (Rev. Fac. Ing.) Vol. 28 (51), pp. 73-87. Abril-Junio 2019. Tunja-Boyacá, Colombia. L-ISSN: 0121-1129, e-ISSN: 2357-5328, DOI: 
the ability to predict physical effects such as frequency dispersion and electronic mobility of the semiconductor device. This is important because the frequency dispersion is one of the most important problems of modern communication systems that generates memory effects limiting the ability to transmit signals of high bandwidth. The fact of being able to predict the electronic mobility and the frequency dispersion help the circuit designer to improve their quality and design time. It also allows the RF component manufacturing industry to save production costs as this technique allows to predict the behavior of the circuits before implementing them. The presented methodology has been validated through the implementation of a power amplifier in LDMOS technology using the proposed technique. The proposed model is open since the proposed new technique can be implemented in any of the conventional models currently used in the industrial and academic field.

Keywords: electronic components; field effect transistors; microwave circuits; microwave measurements; semiconductor device modeling.

\section{Modelo compacto com capacidade de predição de parâmetros físicos para amplificadores de RF}

\section{Resumo}

No presente artigo apresenta-se uma análise de transistores de efeito de campo usando fontes de voltagem pulsadas. Foram realizadas medidas de micro-ondas em dispositivos de tecnologia HEMT's e LDMOS deixando em evidência a diferença entre o comportamento estático e dinâmico de tais dispositivos. Em base às medidas foi realizado um processamento de dados derivando uma nova equação com a capacidade de reproduzir ambos tipos de comportamento com elevada precisão e em diferentes pontos de operação. Consequentemente o trabalho aporta um novo modelo baseado em um circuito não lineal de quatro terminais. A relevância deste modelo é a capacidade de predizer os efeitos físicos como a dispersão frequencial e a mobilidade eletrônica do dispositivo semicondutor. Isto é importante pois a dispersão frequencial é um dos problemas mais relevantes dos sistemas de comunicação modernos que gera efeitos memória limitando a capacidade de transmitir sinais de grande largura de banda. $O$ fato de poder predizer a mobilidade eletrônica e a dispersão frequencial ajudam ao desenhador de circuitos a melhorar sua qualidade e tempo de desenho. Além disso, permite à indústria de fabricação de componentes de RF economizar custos de produção pois esta técnica permite predizer o comportamento dos circuitos antes de implementá-los. A metodologia para a obtenção do modelo compacto tem sido validada através da implementação de um amplificador de potência em tecnologia LDMOS usando a técnica proposta. O modelo proposto é aberto já que a nova técnica proposta pode ser implementada em qualquer dos modelos convencionais usados atualmente no âmbito industrial e acadêmico.

Palavras chave: electronic mobility; field effect transistors; GaAs; microwaves; pulsed measurements; RF power amplifiers.

\section{Para citar este artículo:}

G. Rafael-Valdivia, "Modelo compacto con capacidad de predicción de parámetros físicos para amplificadores de RF," Revista Facultad de Ingeniería, vol. 28 (51), pp. 7387, Abr. 2019. DOI: https://doi.org/10.19053/01211129.v28.n51.2019.9132.

Esta obra está bajo licencia internacional Creative Commons Reconocimiento 4.0 


\section{INTRODUCCIÓN}

Los sistemas de telecomunicación inalámbricos son importantes son importantes en nuestra vida tanto en aplicaciones de internet inalámbrica como en comunicaciones satelitales y comunicaciones celulares. Uno de los elementos más importantes de estos sistemas es el amplificador; y dentro del amplificador son los transistores los componentes principales. Para construir un amplificador es necesario comenzar por una primera etapa denominada la simulación a nivel de transistor. La simulación permite ahorrar tiempo en la etapa de implementación puesto que podemos ver en herramientas CAD el resultado de un amplificador antes de implementarlo físicamente [1].

Dentro de los dispositivos activos de microondas, los transistores HEMT juntamente con el MESFET constituyen los dispositivos unipolares activos de mayor popularidad en los diseños de circuitos de microondas. Fabricantes de gran importancia a nivel mundial como TSMC y Global Foundries están realizando grandes inversiones en el desarrollo de modelos para todos estos tipos de semiconductores.

Un punto crítico en la industria de estos dispositivos lo constituyen los periodos de demora en su fabricación. Para mejorar estos tiempos se disponen de varias técnicas, una de las cuales es el desarrollo de nuevos modelos de mayor precisión que sean capaces de reproducir el comportamiento del dispositivo en diversas condiciones de operación y que se puedan implementar fácilmente en simuladores. De esta manera se puede predecir su comportamiento antes de su fabricación, ahorrando tiempo y dinero.

A raíz de la evolución tecnológica surgieron una diversidad de métodos para el modelado de transistores dentro de los cuales se pueden citar los modelos físicos, los modelos matemáticos, también llamados modelos caja negra (black box) y los modelos de circuito equivalente también denominados compactos.

Los modelos basados en el comportamiento físico plantean las ecuaciones propias del semiconductor y caracterizan el modelo con parámetros físicos como la longitud de puerta, la altura del canal, la densidad del dopado, etc. por esta razón son especialmente útiles en las foundries [2].

Los modelos tipo caja negra, tienen como ventaja la reducción del tiempo de cómputo permitiendo la caracterización del dispositivo a través de un proceso iterativo, sin embargo; no permite identificar parámetros físicos ni eléctricos del comportamiento del transistor. Un ejemplo típico de estos modelos es el que utiliza las Redes Neuronales a través de un algoritmo de aprendizaje con el que se intenta reproducir el comportamiento del transistor [3].

Los modelos eléctricos compactos utilizan un circuito equivalente para simular el comportamiento del dispositivo. Estos modelos constan de un conjunto de elementos lineales y no lineales interconectados. Se han desarrollado varios modelos eléctricos válidos en pequeña señal y gran señal [4]. La ventaja de este tipo de modelado radica en su sencillez y la reducción del tiempo de cómputo. 
La desventaja es que no siempre existe una relación entre los parámetros del modelo eléctrico y los parámetros físicos del dispositivo.

Para lograr superar este reto, en el presente trabajo se analizó el efecto físico de los estados trampa del transistor y su manifestación como la dispersión en frecuencia de la transconductancia y la conductancia de salida. Este último fenómeno produce una variación en la velocidad de los portadores mayoritarios del semiconductor en función del voltaje aplicado, lo cual es conocido como la movilidad electrónica.

En el presente trabajo se plantea como hipótesis que el uso de medidas pulsadas tiene la capacidad de generar información que permite hallar un nuevo modelo de transistores de microondas con la capacidad de predecir no solo los parámetros eléctricos, sino también los fenómenos físicos de transistores en tecnologías GaAs y GaN. En resumen, una ecuación nueva para la fuente de corriente de salida de transistores FET es propuesta.

En la primera sección se detalla la metodología de las medidas, la cual partiendo de extracción de elementos extrínsecos de transistores y de medidas pulsadas nos permite caracterizar el dispositivo en diferentes zonas de funcionamiento. La segunda sección muestra los resultados obtenidos del procesamiento de los datos medidos. En el procesamiento usamos un nuevo circuito equivalente no lineal para dispositivos de microondas, con lo que se obtuvo un modelo compacto. La ventaja del método propuesto es que permite predecir el comportamiento a nivel de dispositivo en cualquier punto de reposo prediciendo a la vez dos fenómenos físicos: la dispersión de la conductancia de salida con respecto al a frecuencia y los cambios en la movilidad electrónica.

Finalmente, en la tercera sección se discute e interpreta los resultados del modelo en términos de precisión y se comparan con los datos medidos. Una característica adicional de este modelo propuesto es que es completamente abierto en el sentido que puede implementarse en cualquier simulador comercial y puede ser combinable con cualquier otro modelo convencional ampliando su capacidad de predicción. Para validar el método propuesto se ha implementado un amplificador de potencia de radiofrecuencia en tecnología LDMOS.

\section{MÉtodos}

El presente trabajo comenzará con la caracterización experimental de transistores de efecto de campo (FET). Para ello se enviarán señales eléctricas de alta frecuencia a cada una de sus terminales: compuerta, drenador y surtidor (representados por G, D y S respectivamente) con el objetivo de medir sus elementos de acceso, también conocidos como elementos extrínsecos 0 parásitos. Posteriormente se desarrollará el modelado de dichos transistores. Para ello tomaremos en cuenta los valores de los elementos parásitos obtenidos en la caracterización. Finalmente, para validar los resultados se presentará la implementación de amplificadores usando los resultados de la técnica de modelado propuesta. 


\section{A. Método de extracción de elementos parásitos}

El procedimiento convencional para extraer elementos parásitos de transistores de microondas contempla el uso de medidas en FET "frío", vale decir con un voltaje de drenador igual a cero voltios. Sin embargo; este tipo de procedimiento puede presentar ciertos inconvenientes de precisión en puntos de reposo diferentes al FET "frío".

En este artículo se utiliza la extracción de elementos parásitos usando la configuración de FET en "caliente" en condiciones multibias. La técnica de FET "frío" utiliza métodos matemáticos convencionales de optimización para calcular los valores de los elementos parásitos; pero en ocasiones los resultados obtenidos quedan lejos de la solución correcta. En el caso de extracción de parásitos en configuración FET "caliente", presentado en este artículo, se usa la técnica de inteligencia artificial propuesta en [5] para la optimización multibias la cual toma en cuenta el conocimiento del ingeniero de diseño en el sentido que permite optimizar solo los parásitos necesarios para su ajuste con una determinada medida experimental.

Nuestro primer vehículo de prueba es el transistor NE76038 de NEC Semiconductors. Pertenece a la familia de los FET de hetero-unión pseudomórficos. El circuito equivalente en pequeña señal [6] usado para representar este dispositivo está detallado en la Figura 1. Todos los elementos que están fuera del rectángulo con línea punteada son los elementos parásitos. Como se puede apreciar son 8 elementos parásitos. En este artículo consideramos que un elemento parásito no cambia su valor con respecto al punto de polarización, vale decir que sus valores son válidos en cualquier punto de polarización del transistor.

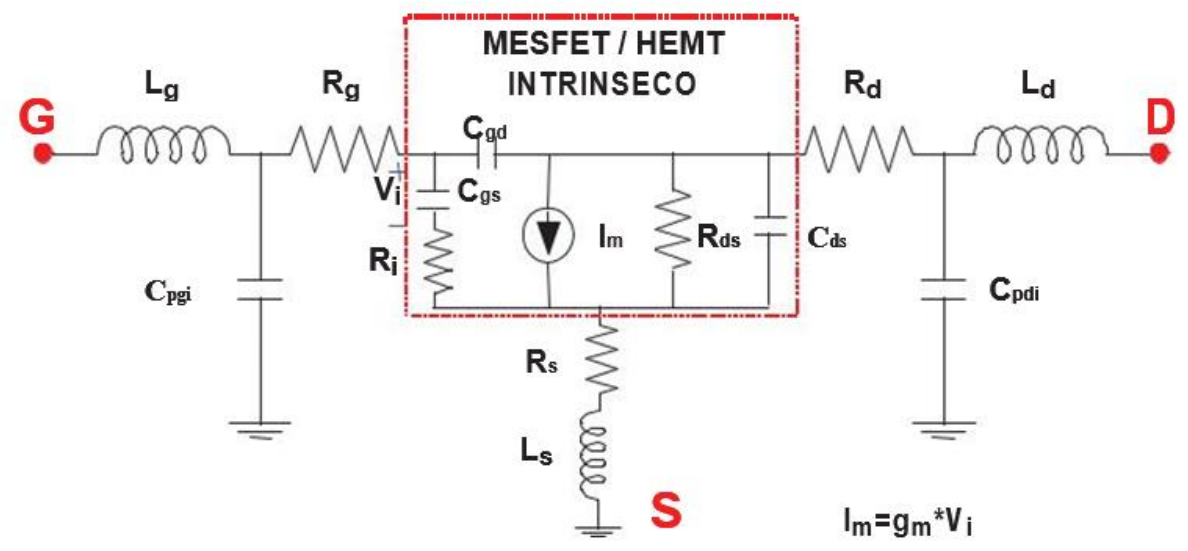

Fig. 1. Circuito eléctrico equivalente en pequeña señal para transistores de alta frecuencia.

\section{B. Método de extracción de Modelo Lineal y no lineal}

Una vez extraídos los elementos parásitos, se usa el método de medidas pulsadas con sistema automatizado DIVA de Accent Technologies [7], mostrado en la siguiente figura. El sistema DIVA permite enviar y recibir pulsos de voltajes por cada uno de sus dos terminales. Estos son aplicados a la entrada y salida del transistor (compuerta y drenador respectivamente) el cual se halla montado dentro de un test fixture como se muestra en la Figura 2. 


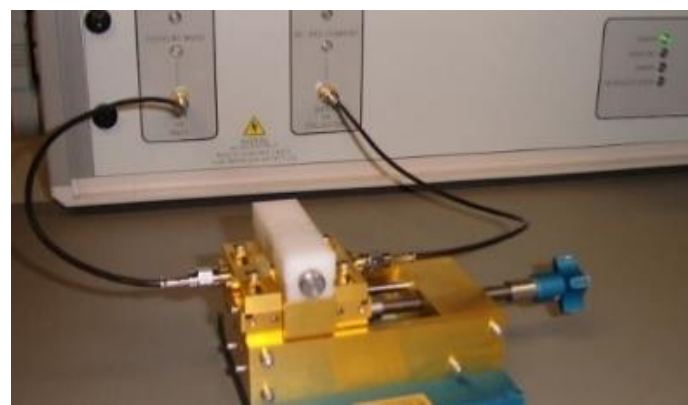

Fig. 2. Sistema de medida DIVA usado para caracterizar los efectos dinámicos de transistores de microondas. El transistor a medir se halla debajo del pusher de plástico blanco ubicado en el medio del test fixture.

Los voltajes enviados son configurados en forma de pulsos considerando un ancho de $0.2 \mu$ segundos y un periodo de un milisegundo. Esto se hizo con el propósito de medir únicamente el comportamiento dinámico manteniendo constante el mismo punto de reposo. El sistema nos permite además medir las características DC IV y compararlas con las características pulsadas IV del transistor.

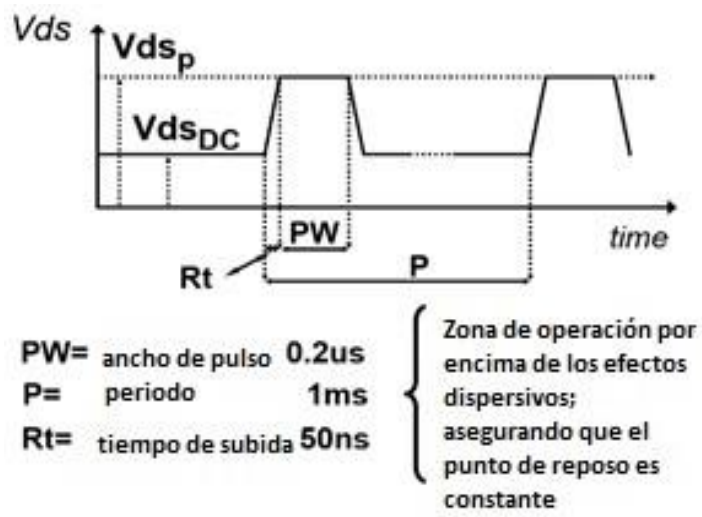

Fig. 3. Formas de onda del voltaje enviado hacia el drenador por el sistema DIVA.

El resultado de esta medida es una matriz de 4 columnas que almacena los valores de las corrientes de drenador y de compuerta (Ids e Igs respectivamente) para las diferentes combinaciones de los voltajes aplicados (Vgs y Vds). Para procesar los datos anteriores, se usa la técnica mostrada en [8], donde se hace uso de los denominados "voltajes efectivos", mostrados en (1) y (2).

$$
\begin{aligned}
& \mathrm{vdSe}=\mathrm{dvd}_{\mathrm{vd}} \cdot\left(\mathrm{k}_{1}-\mathrm{k}_{2} \cdot \mathrm{d}_{\mathrm{vd}}\right)+\mathrm{Vdi}_{\mathrm{DC}}+\mathrm{d}_{\mathrm{vg}} \cdot\left(\mathrm{k}_{3}-\mathrm{k}_{4} \cdot \mathrm{d}_{\mathrm{vg}}\right) \\
& \mathrm{vgSe}=\mathrm{dvg}_{\mathrm{vg}} \cdot\left(\mathrm{k}_{5}-\mathrm{k}_{6} \cdot \mathrm{dvg}_{\mathrm{vg}}\right)+\mathrm{Vgi}_{\mathrm{DC}}+\mathrm{dvd}_{\mathrm{vd}} \cdot\left(\mathrm{k}_{7}-\mathrm{k}_{8} \cdot \mathrm{d}_{\mathrm{vd}}\right)
\end{aligned}
$$

Donde:

vdse, vgse representan los voltajes efectivos en drenador y surtidor.

$d_{v g}$, dvd representan la diferencia entre voltajes pulsados y DC aplicados en puerta (Vgsp - Vgsdc) y en drenador (Vdsp - Vdsdc) respectivamente.

$\mathrm{k}_{1}$ to $\mathrm{k}_{8}$ representan parámetros de optimización.

Vdsp $V_{g s p}$ representan las amplitudes de los voltajes pulsados. 
Las magnitudes obtenidas vdse y vgse son anidadas dentro de la ecuación de COBRA [9] resultando una ecuación de 4 dimensiones para lds.

$$
\operatorname{Ids}=\mathrm{F}\left(\operatorname{Vdi}_{\mathrm{p}}, \operatorname{Vgi}_{\mathrm{p}}, \operatorname{Vdi}_{\mathrm{DC}}, \operatorname{Vgi}_{\mathrm{DC}}\right)
$$

Para demostrar la efectividad del método mencionado, se hace un programa en Matlab donde se optimizan los parámetros " $k$ " de la nueva ecuación con los ficheros de las medidas pulsadas. Los resultados que se mostrarán en la siguiente sección demostrarán la capacidad predictiva de la ecuación propuesta. Las variables vdse y vgse también fueron anidadas dentro de otra ecuación (denominada Angelov) propuesta en [10] con similares resultados. En consecuencia, es posible demostrar que la técnica de anidar los voltajes efectivos dentro de una ecuación empírica puede aplicarse con diferentes ecuaciones convencionales, ampliando su rango de aplicación dotándole de la capacidad de predecir medidas pulsadas.

La ecuación propuesta con los parámetros "k" optimizados fue implementada así mismo en un componente SDD del software de simulación ADS. Este componente fue asociado a una fuente de corriente no lineal denotada por Ids (corriente de salida), la cual junto con las capacitancias no lineales Cgd y Cgs forman el circuito equivalente no lineal de nuestro transistor.

En la Figura 4 se representa dicho circuito donde se aprecia en la parte izquierda el terminal de entrada (compuerta) y a la derecha el terminal de salida (drenador). Es importante indicar que dicho circuito representa el equivalente intrínseco y que los elementos extrínsecos (resistencias, inductancias y capacitancias parásitas) no han sido dibujados por simplicidad, pero sus valores (extraídos en la primera sección) han sido considerados a la hora de hacer las medidas para poder obtener los voltajes y corrientes intrínsecos del transistor.

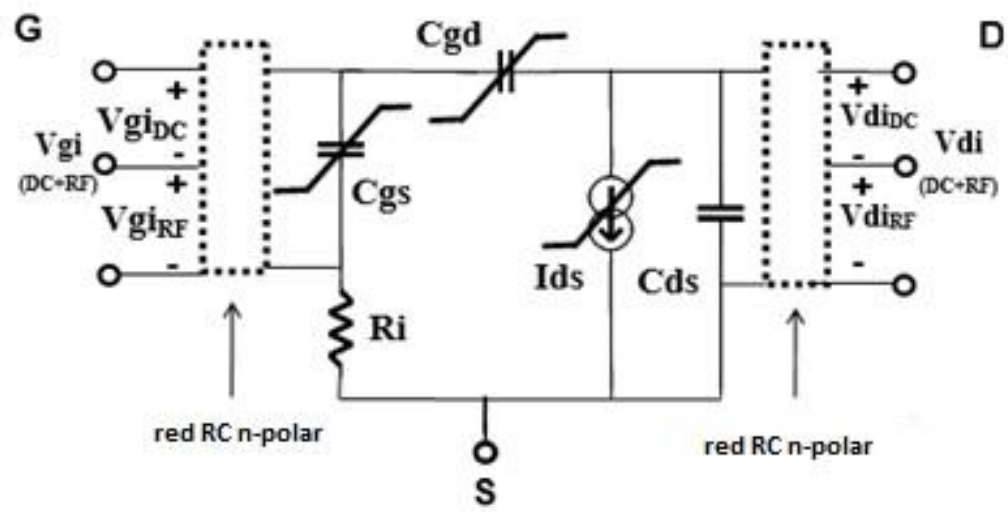

Fig. 4. Circuito no lineal gran señal empleado.

En el circuito equivalente no lineal de la Figura 4 [11] se observa la presencia de una red $\mathrm{RC}$ multipolo tanto en el drenador como en la compuerta. Estas han sido diseñadas para separar los componentes estáticos y dinámicos del sistema.

Aplicando medidas de baja frecuencia en nuestro transistor, las redes multipolo fueron sintetizadas como se muestra en la Figura 5. 


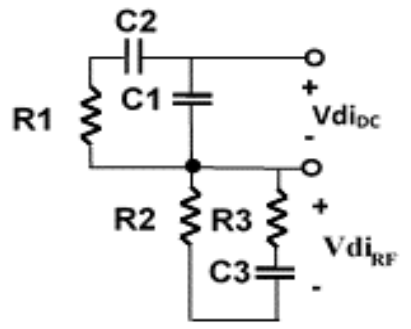

Fig. 5. Red RC n-polar sintetizada.

Extrayendo las derivadas de la Ecuación 3, es posible hallar expresiones analíticas para modelar la conductancia de salida y la transconductancia, tanto en baja como en alta frecuencia:

$$
\frac{\partial \mathrm{Ids}}{\partial \mathrm{Vgi} \mathrm{RF}_{\mathrm{mRF}}}=\frac{\partial \mathrm{Ids}}{\partial \mathrm{VgidC}}=\boldsymbol{g}_{\mathrm{mDC}} ; \frac{\partial \mathrm{Ids}}{\partial \mathrm{VdiRF}}=\boldsymbol{g}_{\mathrm{dRF}} ; \frac{\partial \mathrm{Ids}}{\partial \mathrm{VdidC}}=\boldsymbol{g}_{\mathrm{dDC}}
$$

La performance del modelo en términos de dispersión en frecuencia se muestra en la Figura 6.

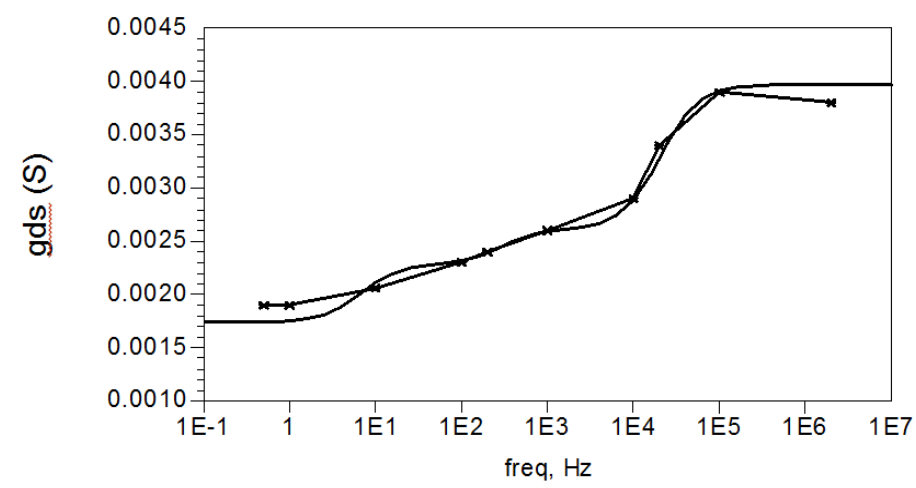

Fig. 6. Dispersión frecuencial de la conductancia de salida (gds): medida ( $\mathrm{x}$ ) y modelada (línea contínua) evaluadas en Vds ${ }_{\mathrm{DC}}=3 \mathrm{~V}$; Vgs $\mathrm{S}_{\mathrm{DC}}=0 \mathrm{~V}$ para el NE76038.

Una vez implementado este circuito en el simulador, se procede a hacer una simulación de parámetros de Scattering. Estos se comparan con medidas hechas en VNA a efectos de validación. Posteriormente se procede a extraer la movilidad electrónica y a modelar los efectos trampa a partir del modelo propuesto. Los resultados se mostrarán en la siguiente sección.

\section{Método de diseño de amplificador de potencia}

Usando los resultados anteriores es posible pasar desde un análisis a "nivel de transistor" hacia "nivel de amplificador". Para ello se utiliza la metodología de extracción de parásitos y modelado no lineal propuestos anteriormente utilizando un transistor de potencia: el MW7IC3825GN, el cual es un dispositivo dedicado a aplicaciones de estación base para telefonía celular con una banda de frecuencia de 3.4 a $3.6 \mathrm{GHz}$ [12]. Para anticipar los resultados en la fase de diseño se usan los modelos de ROOT y de MET [13], típicos de esta tecnología. Si las metodologías expuestas en las secciones A y B son coherentes, se espera obtener un amplificador que satisfaga los requerimientos de usuario con valores precisos de impedancias óptimas. Los resultados numéricos se mostrarán en la sección siguiente. 


\section{Resultados}

Para validar nuestra metodología, usamos un dispositivo de baja potencia: el NE76038 y otro de alta potencia: el MW7IC3825GN. Aplicando el método de extracción de parásitos propuesto en los apartados A y B al transistor NE76038 de NEC Semiconductors se obtuvieron los resultados de la Tabla I.

Tabla I. Elementos parásitos extraídos para el dispositivo bajo prueba.

\begin{tabular}{|c|c|c|c|c|c|c|c|}
\hline \multicolumn{2}{|c|}{ RESISTENCIAS (ohms) } & \multicolumn{3}{c|}{ INDUCTANCIAS (nH) } & \multicolumn{2}{c|}{ CAPACIDADES (pF) } \\
\hline $\mathrm{Rg}$ & $\mathrm{Rd}$ & $\mathrm{Rs}$ & $\mathrm{Lg}$ & $\mathrm{Ld}$ & $\mathrm{Ls}$ & Cpgi & Cpdi \\
\hline 3.35 & 2.88 & 0.06 & 0.241 & 0.863 & 0.214 & 0.141 & 0.140 \\
\hline
\end{tabular}

Posteriormente, usando el sistema de medidas pulsadas DIVA se recolectaron datos de corriente pulsada (Ids) los cuales fueron usados para optimizar la ecuación de 4 variables señalada en el apartado C. Tal como se observa en la Figura 7 se obtuvo una excelente similitud entre datos medidos y simulados.
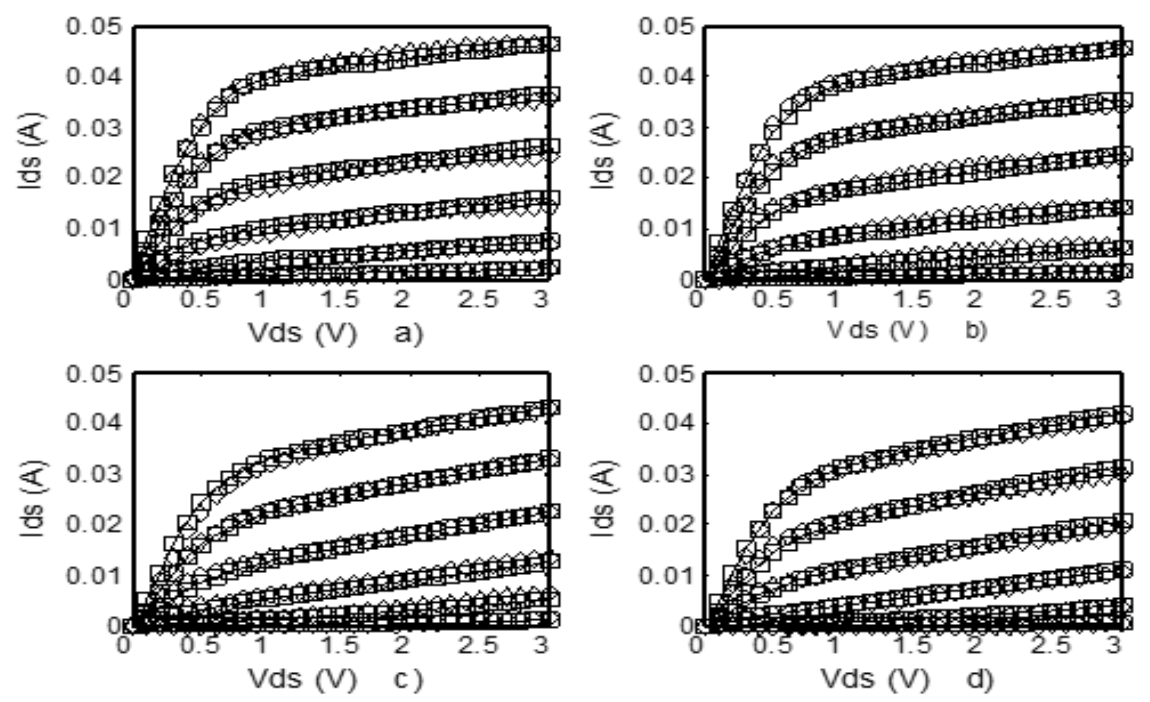

Fig. 7. Ids pulsados: Medidas (rombos) y simulados (cuadrados). Ancho del pulso: $0.2 \mu \mathrm{sec}$; Duty cycle: $0.02 \%$. Voltajes aplicados: a) $\mathrm{VgS}_{\mathrm{DC}}=0 \mathrm{~V}, \mathrm{Vds}_{\mathrm{DC}}=0.5 \mathrm{~V}$; b) $\mathrm{VgS}_{\mathrm{DC}}=-$ $1 \mathrm{~V}, \mathrm{Vds}_{\mathrm{DC}}=0.5 \mathrm{~V}$; $\left.\mathrm{c}\right) \mathrm{Vg} \mathrm{s}_{\mathrm{DC}}=0 \mathrm{~V}, \mathrm{Vds_{DC }}=3 \mathrm{~V}$; d) $\mathrm{Vgs}_{\mathrm{DC}}=-1 \mathrm{~V}, \mathrm{Vds_{DC }}=3 \mathrm{~V}$.

Para validar nuestro modelo propuesto, se hicieron otro tipo de medidas usando un VNA. Los resultados medidos de parámetros $S$ en el punto de reposo $V d S_{D C}=3 \bigvee$ y $V_{g S D C}=0 V$ se muestran en la Figura 8. Los valores obtenidos a partir del modelo propuesto en ADS coinciden con los valores obtenidos de las medidas de VNA, lo que revela la coherencia del modelo. Además, haciendo un barrido en potencia se puede demostrar que dicho modelo es capaz de predecir no solamente la componente fundamental sino también las componentes de intermodulación, [11] tal como se muestra en la Figura 9. 


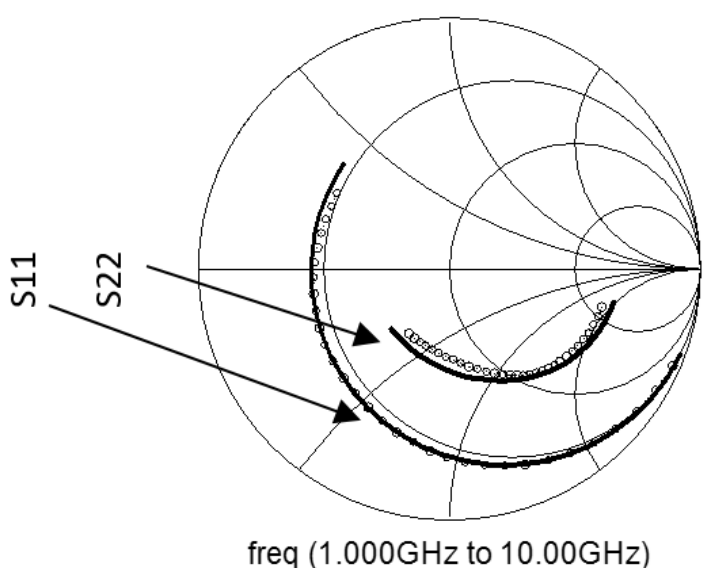

Fig. 8. Parámetros S medidos (círculos) y modelados (líneas).

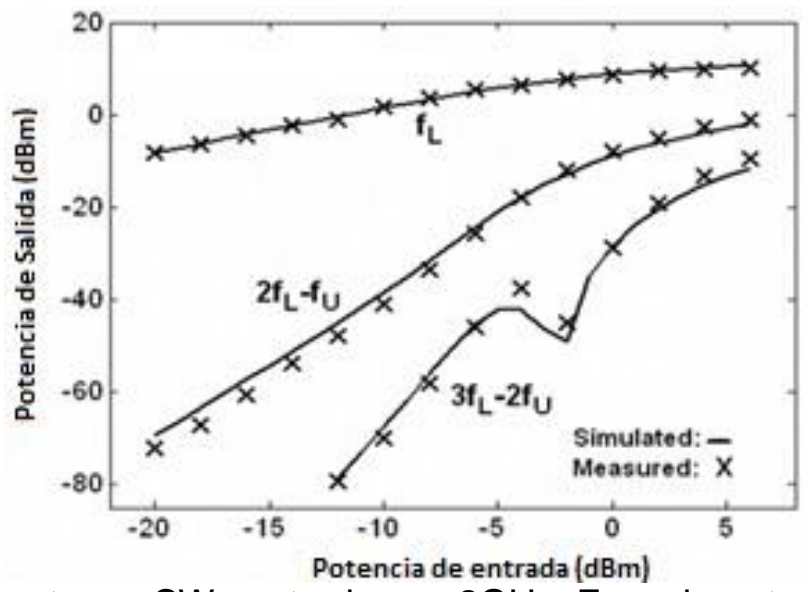

Fig. 9. Test de dos tonos $\mathrm{CW}$ centrados en $2 \mathrm{GHz}$. Espacio entre tonos: $4 \mathrm{MHz}$. $\mathrm{Vds} \mathrm{SC}_{\mathrm{C}}=2 \mathrm{~V} ; \mathrm{VgS}_{\mathrm{DC}}=-0.2 \mathrm{~V}$.

Con el objetivo de demostrar la coherencia física del modelo propuesto, se procedió a modelar y extraer la movilidad electrónica del transistor. Para ello, se usó el método de las derivadas de orden superior [14] el cual permite calcular la movilidad a partir del parámetro "gmd" (segunda derivada de Ids). El parámetro "gmd" fue extraído analíticamente derivando la ecuación propuesta de Ids usando Matlab. Los resultados obtenidos permiten verificar que para el punto de operación VDS $=0.15 \mathrm{~V}, \mathrm{VGS}=-0.3 \mathrm{~V}$, considerando la longitud de puerta $\mathrm{L}=0.3 \mu \mathrm{m}$ y habiendo extraído $\mathrm{Cg}=0.1 \mathrm{pF}$, y $\mathrm{gmd}=100 \mathrm{mS} / \mathrm{V}$, se obtiene un valor teórico modelado de movilidad de $900 \mathrm{~cm}^{2} /$ (V.s).

Para comparar este resultado modelado con valores experimentales se procedió a medir la movilidad. Para ello se usó el VNA tomando medidas de los parámetros $S$ para diferentes valores de VGS. A continuación, se procedió a hacer la extracción de gm, Cg y movilidad, para representarlos con respecto a la frecuencia. Los resultados obtenidos se muestran en las Figuras 10, 11 y 12. 


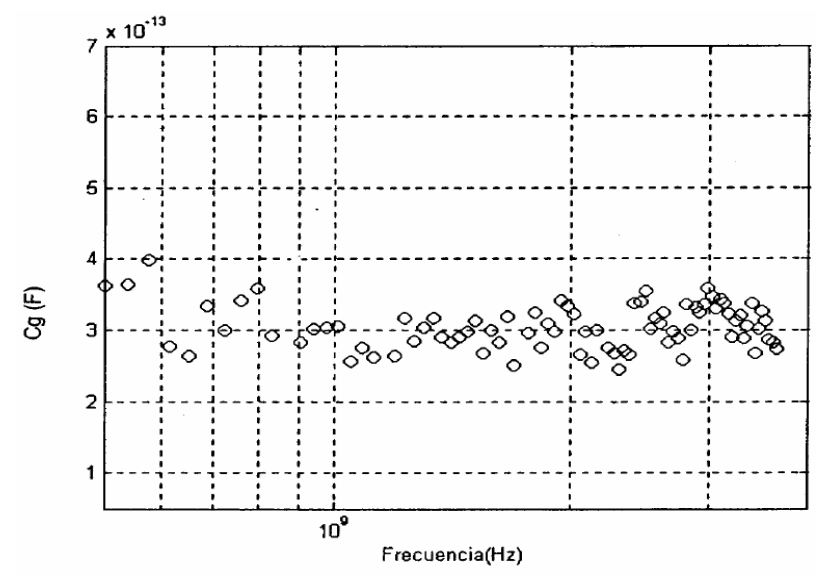

Fig. 10. Valores de Cg extraídos a partir de los parámetros $S$ en el punto de polarización VGS $=0 \mathrm{~V}, \mathrm{VDS}=0.2 \mathrm{~V}$.

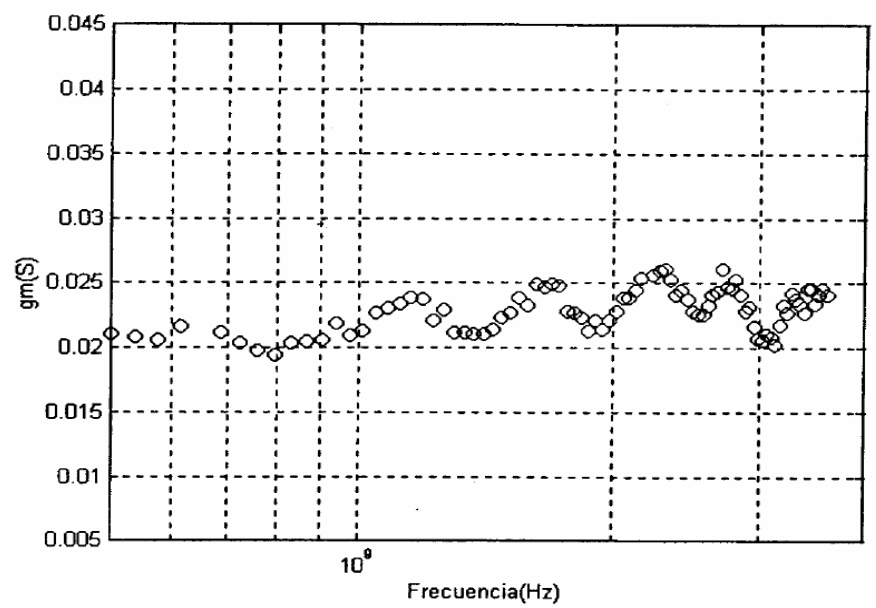

Fig. 11. Valores obtenidos de gm a partir de los parámetros $S$ en el punto de polarización VGS $=0 \mathrm{~V}, \mathrm{VDS}=0.2 \mathrm{~V}$.

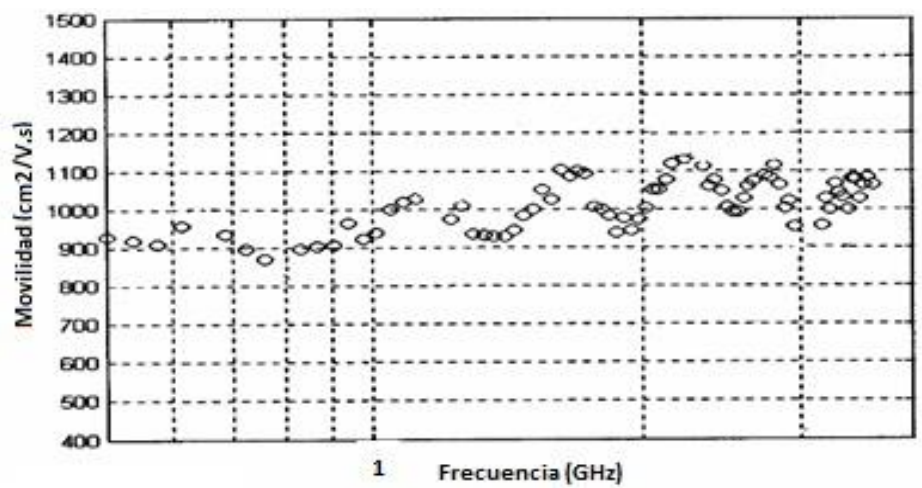

Fig. 12. Medidas experimentales de movilidad respecto a la frecuencia extraídas a partir de los parámetros $\mathrm{S}$.

La Figura 12 muestra los valores medidos de movilidad alrededor de 1000 $\mathrm{cm}^{2} /$ V.s. Estos valores son similares dentro de un $10 \%$ a los calculados teóricamente a partir del modelo propuesto $\left(900 \mathrm{~cm}^{2} / \mathrm{V} . \mathrm{s}\right)$. La variación y la tendencia a aumentar puede atribuirse a los efectos trampa y a la dispersión de alta frecuencia del semiconductor. 
Una vez validado el modelo a nivel de componente, se procedió a la validación a nivel de circuito. Para ello se hicieron medidas de load pull a nivel de transistor descontando los efectos de los elementos parásitos señalados anteriormente. El transistor usado fue el MW7IC3825GN. Los resultados se muestran en la Tabla II. Como resultado de las medidas, el dispositivo mostró tener potencia, ganancia y eficiencia planas en un gran ancho de banda.

Tabla II. Resultados de medidas de load pull a nivel de transistor.

\begin{tabular}{|c|c|c|c|c|c|}
\hline Freq.(GHz) & Zload(Ohms) & P1dB(dBm) & Gain(dB) & IRL(dB) & Efficiency(\%) \\
\hline 3.3 & $4.61-j .10 .56$ & 46.23 & 23.6 & -23 & 41.4 \\
\hline 3.4 & $4.55-\mathrm{j} .8 .49$ & 46.30 & 24.7 & -24 & 43.0 \\
\hline 3.6 & $3.26-j .8 .49$ & 46.23 & 23.5 & -29 & 41.9 \\
\hline 3.8 & $3.08-\mathrm{j} 10.33$ & 46.26 & 21.3 & -38 & 40.0 \\
\hline
\end{tabular}

Antes de implementar el amplificador se realizó una simulación. Para ello se usó la metodología de voltajes efectivos, señalada en la sección anterior con dos modelos diferentes: ROOT y MET. Los resultados se presentan en la Tabla III, donde se puede ver que el modelo MET tiene una precisión de 0.5ohms en cuanto a la predicción de las impedancias óptimas.

Tabla III. Comparación entre impedancias medidas y modeladas.

\begin{tabular}{|c|c|c|c|c|}
\hline \multirow{2}{*}{$\begin{array}{c}\text { 3.6GHz } \\
\text { MET }\end{array}$} & \multicolumn{2}{|c|}{ Max Pout @1dB } & \multicolumn{2}{c|}{ Max PAE @1dB } \\
\cline { 2 - 5 } & Medida & Modelo & Medida & Modelo \\
\hline Zout(Ohm) & $3.3-j .8 .3$ & $3.9-j .8 .3$ & $3.5-j .7 .1$ & $3.6-j .7 .7$ \\
\hline $\begin{array}{c}\text { 3.6GHz } \\
\text { ROOT }\end{array}$ & \multicolumn{2}{|c|}{ Max Pout @1dB } & \multicolumn{2}{c|}{ Max PAE @1dB } \\
\cline { 2 - 5 } & Modelo & Modelo & Medida & Modelo \\
\hline Zout(Ohm) & $3.3-j .8 .3$ & $4.3-j .10 .1$ & $3.5-j .7 .1$ & $4.2-j .7 .8$ \\
\hline
\end{tabular}

Finalmente se implementó un amplificador demostrativo usando el substrato comercial Taconic RF35 de bajas pérdidas. La red de polarización usó líneas y transformadores de cuarto de onda con el fin de minimizar el uso de componentes discretos tal como se muestra en la Figura 13. Polarizando el dispositivo en condiciones adecuadas se obtuvo una ganancia aproximada de $24 \mathrm{~dB}$ desde 3.4 a $3.6 \mathrm{GHz}$. Realizando barrido en potencia se pudo verificar que la potencia de salida a $1 \mathrm{~dB}$ de compresión era de $29 \mathrm{~W}$.

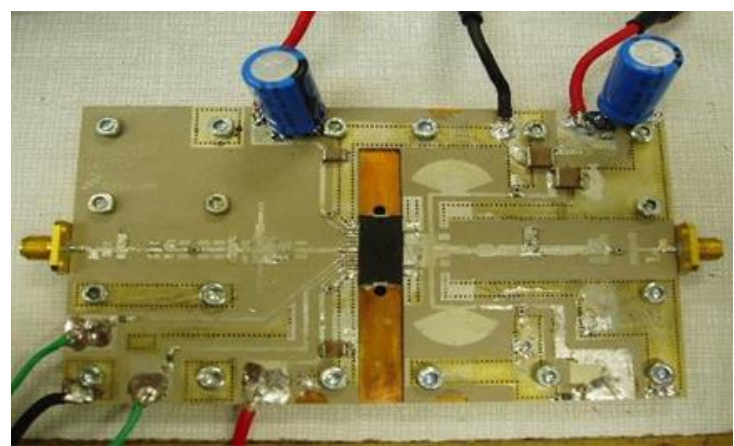

Fig. 13. Amplificador de potencia implementado con el MW7IC3825GN. 


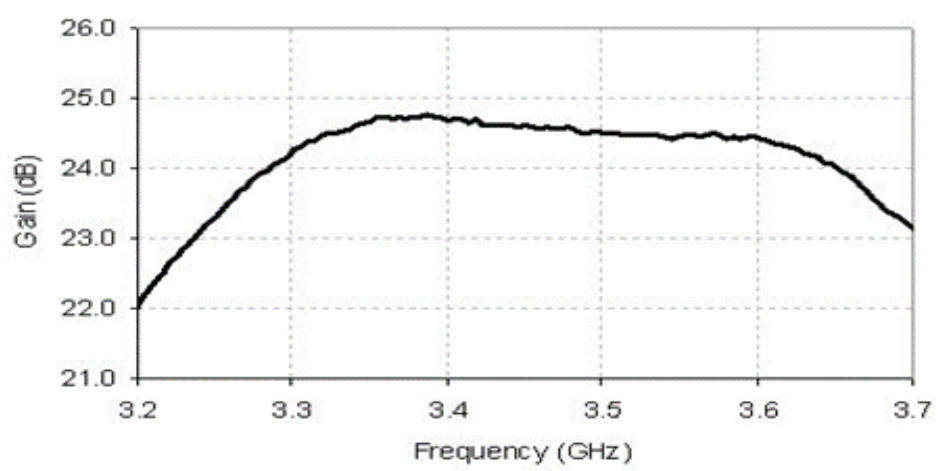

Fig. 14. Respuesta en frecuencia del amplificador implementado.

\section{DiscuSIÓN Y CONCLUSIONES}

En el presente trabajo se ha propuesto una nueva metodología para la implementación de modelos compactos para amplificadores de microondas basado en medidas pulsadas. En una primera parte se ha planteado el uso de la inteligencia artificial en el proceso de optimización para extraer los elementos parásitos de un transistor, obteniéndose resultados de alta precisión. En la segunda parte se ha mostrado que el método de modelado compacto no lineal usando medidas pulsadas permite predecir con precisión el comportamiento del transistor en condiciones estáticas y dinámicas. El método propuesto usa la metodología de voltajes efectivos la cual tiene como valor añadido que permite predecir fenómenos físicos del transistor como son la movilidad electrónica y la dispersión de la conductancia de salida. Finalmente se ha validado la metodología implementando un amplificador de potencia de RF. Se ha demostrado que usando el método propuesto para la extracción de modelos compactos es posible obtener las impedancias óptimas que permiten satisfacer las especificaciones técnicas de ganancia y potencia deseadas en amplificadores de microondas.

\section{REFERENCIAS}

[1] A. Eroglu, Introduction to RF Power Amplifier Design and Simulation, $1^{\text {st }}$ Edition, NY, CRC Press, Jul. 2015.

[2] S. M. Sze, Physics of Semiconductor Devices. Bell Laboratories, $3^{\text {rd }}$ Edition. Hoboken, NJ, USA, John Wiley \& Sons Inc., Oct. 2006.

[3] C. Crespo-Cadenas, J. Reina, and M. Madero, "A Volterra-Based Procedure for Multi-Port and MultiZone GaN FET Amplifier CAD Simulation," Transactions on circuits and systems, vol. 60 (11), pp. 3022-3032, Nov. 2013. DOI: https://doi.org/10.1109/tcsi.2013.2252691.

[4] T. Fernández, Y. Newport, J. M. Zamanillo, A. Tazón, and A. Mediavilla, "Modelling of Operating Point Non Linear Dependence of Ids Characteristics from Pulsed Measurements in MESFET transistors," In 23 Eu.M.C., p. 518, Sep. 1993. DOI: https://doi.org/10.1109/euma.1993.336612.

[5] G. Rafael Valdivia, J. M. Zamanillo, C. Pérez-Vega, T. Fernández, A. Mediavilla, and A. Tazón. "Método neurodifuso para la extracción de los elementos parásitos en MESFET y HEMT," In: XVII Simposium Nacional de la Unión Científica Internacional de Radio, Alcalá de Henares, 2002.

[6] G. Dambrine, A. Cappy, F. Heliodore, and E. Playez, "A New Method for determining the FET smallsignal equivalent circuit," IEEE Transactions on Microwave Theory and Techniques, vol. 36 (7), pp. 1151-1159, Jul. 1988. DOI: https://doi.org/10.1109/22.3650.

[7] T. Hackbarth, H.-J. Herzog, K.-H. Hieber, U. Konig, S. Mantl, B. Hollander, S. Lenk, and H. von Kanel, "Strained silicon FETs on thin SiGe virtual substrates produced by He implantation: reduced selfheating on DC and RF performance," In: International Semiconductor Device Research Symposium, 2003. DOI: https://doi.org/10.1109/isdrs.2003.1271992. 
[8] G. Rafael-Valdivia, R. Brady, and T. Brazil, "Single function drain current model for MESFET/HEMT devices including pulsed dynamic behavior," In: IEEE MTT-S International Microwave Symposium Digest, Nov. 2006. DOI: https://doi.org/10.1109/mwsym.2006.249595.

[9] V. Cojocaru, and T. Brazil, "A Scalable General-Purpose Model for microwave FET's Including DC/AC dispersion effects," IEEE Trans. on Microwave Theory and Techniques. vol. 45 (12), pp. 22482255, Dec. 1997. DOI: https://doi.org/10.1109/22.643826.

[10] I. Angelov, H. Zirath, and N. Rorsman, "A new empirical nonlinear model for HEMT-devices," In: IEEE MTT-S Microwave Symposium Digest, 1992. DOI: https://doi.org/10.1109/mwsym.1992.188320.

[11] G. Rafael-Valdivia, and Z. Su, "Non-linear modeling for low and high power microwave transistors," In: 46th European Microwave Conference (EuMC), 2016. DOI: https://doi.org/10.1109/eumc.2016.7824476.

[12] C. Cassan, and G. Rafael-Valdivia. "Two-Stage LDMOS RFIC Drives WiMAX," Microwaves \& RF Journal, vol. 47(10), pp. 87-97, Oct. 2008.

[13] J. A. Pla, and D. Bridges, "A robust high voltage Si LDMOS model extraction process to achieve first pass linear RFIC amplifier design success," In: IEEE MTT-S International Microwave Symposium Digest, 2002. DOI: https://doi.org/10.1109/mwsym.2002.1011607.

[14] G. Rafael-Valdivia, T. Férnández-lbáñez, J. Rodríguez-Tellez, A. Tazón Puente, and A. MediavillaSánchez, "Measurement of Mobility in HEMT Devices Using High-Order Derivatives," IEEE Transactions on Electron Devices, vol. 51 (1), pp. 1-7, Jan. 2004. DOI: https://doi.org/10.1109/ted.2003.820938. 